\title{
A study on radiological outcomes of accelerometer based hand held navigation system in total knee arthroplasty
}

\author{
Hiranyakumar S., Shivaraj Nadagouda, Vishnu Harikrishnan*
}

\begin{abstract}
Department of Orthopedics, Vydehi Institute of Medical Sciences and Research Centre, Whitefield, Bangalore, Karnataka, India
\end{abstract}

Received: 19 January 2020

Revised: 27 February 2020

Accepted: 28 February 2020

\author{
*Correspondence: \\ Dr. Vishnu Harikrishnan, \\ E-mail: vishnuharikrishnan1210@gmail.com
}

Copyright: () the author(s), publisher and licensee Medip Academy. This is an open-access article distributed under the terms of the Creative Commons Attribution Non-Commercial License, which permits unrestricted non-commercial use, distribution, and reproduction in any medium, provided the original work is properly cited.

\begin{abstract}
Background: The goal of obtaining more accurate component alignment in total knee arthroplasty (TKA) has led to the development of techniques like portable accelerometer-based handheld navigation system, computer assisted system, patient specific instrumentation. Among which hand held navigation has showed promising results for achieving accurate resection and alignment and only few studies were done to assess radiological outcome or the effective component alignment using portable accelerometer-based hand held navigation system in TKA.

Methods: TKA using portable accelerometer-based hand held navigation system (Smith and Nephew- knee align) was done on 25 knees with primary osteoarthritis. Preoperative and post-operative mechanical axis and component alignments were measured using hip-to-ankle radiographs.

Results: Among the femoral components, $92.0 \%$ were placed within $90^{\circ} \pm 2^{\circ}$ to the femoral mechanical axis in the coronal and $96.0 \%$ of the tibial components were placed within $90^{\circ} \pm 2^{\circ}$ to the mechanical axis in the coronal plane, $94.3 \%$ of the TKAs had an overall lower extremity alignment within $3^{\circ}$ of neutral to the mechanical axis, based on postoperative hip-to-ankle radiographs.

Conclusions: The degree of accuracy in positioning the femoral and tibial component in TKA using portable accelerometer-based hand held navigation system (knee align) is very much promising and is an effective intraoperative tool for reducing the proportion of outliers for component placement and thus decreasing the chances of instability and early wear and tear of the components in TKA.
\end{abstract}

Keywords: Knee, Arthroplasty, Femoral component alignment, Tibial component alignment, Hand held navigation

\section{INTRODUCTION}

Total knee arthroplasty (TKA) is primarily done to relieve the pain caused by severe arthritis with or without significant deformity. ${ }^{1}$ Aseptic loosening is an increasingly frequent cause of TKA failure that requires revision and one of the main causes of instability is malalignment. $^{2}$

The goal of obtaining more accurate component alignment in TKA has led to the development of various techniques like portable accelerometer-based handheld navigation system, computer assisted system (CAS), patient specific instrumentation (PSI). When PSI is compared to conventional TKA there were no significant difference in the radiographic outcome and moreover the operating time was more. In CAS the cost, operating time, chances of periprosthetic fractures are very high. ${ }^{3,4,5}$

The need to overcome these limitations led to the development of hand held navigation system. It has showed promising results for achieving accurate tibial and femoral resection and alignment as with the same degree of accuracy as with large console CAS systems, while 
preventing the above mentioned concern. ${ }^{5}$ It also does not depend on assumptions based on the axes of the femur and tibia like in conventional method. ${ }^{6}$

I am doing this study, as not many studies have been done on the radiological outcome or the effective component alignment using portable accelerometer-based hand held navigation system in TKA. The aim of this study is to assess radiologically the accuracy of femoral resection within 3 degree of perpendicular to the femoral mechanical axis, the accuracy of tibial resection within 3 degree of perpendicular to the tibial mechanical axis and the overall mechanical alignment.

\section{METHODS}

Between January and June 2018, a total of 23 consecutive patients with primary osteoarthritis of the knee were prospectively selected for TKA using hand held navigation system to perform femoral and tibial resection. This study is done in the department of orthopaedics, Vydehi Institute of Medical Sciences and Research Centre, Whitefield, Bangalore, India.

All patients received a total knee arthroplasty from the same surgeon with experience in knee arthroplasty surgery. Patients were included in the study if they were a case of primary osteoarthritis. The mean age of patients included in the study was 62.5 years (range 45-80 years). Patients were excluded if they had bony defects which require the usage of metallic wedges and stem extenders, arthritis secondary to infection or if it is case of revision arthroplasty.

All patients gave written informed consent prior to participation in the study, which was approved by the institutional ethics committee. Routine investigations needed prior to surgery was done like blood and urine investigations: $\mathrm{Hb} \%$, TC, DC, ESR, RFT, LFT, lipid profile, FBS/PPBS, HIV, HBsAg, urine for albumin, sugars, ketone bodies and microscopy, X-ray of chest, ECG, 2D echo.

X-ray imaging pre- and post-operative standing AP hip-toankle radiographs and standing lateral knee radiographs. Patients received a posterior-cruciate ligament substituting TKA. Tibial and femoral resection was done using the Ortho Align system. Among the 23 patients 2 cases underwent bilateral TKA in which the second surgery was done 2 weeks after the first one.

\section{Surgical technique}

The knee align navigation system is a palm-sized unit used to assist the operating surgeon with coronal (varus/valgus), sagittal (posterior slope) tibial component and femoral component varus/valgus positioning. The navigation system is a handheld accelerometer-based surgical navigation system which consists of a display console, a reference sensor, a femoral jig and tibial jig (Figure 1).
The femoral jig is placed on the distal femoral condyles, with its midpoint centered at the deepest point of the intercondylar notch. The femoral jig is fixed to the distal femur using three $3.2 \mathrm{~mm}$ threaded pins. The display console is attached to the femoral jig, and the reference sensor is attached to the distal femoral cutting block (Figure 2). The initial position of the cutting block is registered first.

Next, the hip centre of rotation is registered by moving the femur up and down and mediolaterally as directed by the display console (requires 2-3 cycles). After the hip centre of rotation and the initial cutting block position are known, the knee align is ready to assess and determine the cutting block's position in both the coronal (varus/valgus) and sagittal (flexion/extension) planes.

The depth of resection is adjusted by moving the cutting block proximal or distal with respect to the distal femoral condyles. Once the operating surgeon has confirmed the cutting block's position, it is pinned to the anterior femur using 2 headless pins, and then the distal femoral resection is performed. Next is the preparation of tibia. There are 2 primary components of tibial jig- the mobile component and a fixed component. The fixed component is attached to the bone and mobile component guides the cutting block.

The display unit is attached to the mobile component and the reference sensor is attached to the fixed component of the jig (Figure 3). This is to compensate for the leg movements. The tibial jig is pinned onto the tibial tubercle, and the mechanical axis of the tibia is established.

The medial and lateral malleoli are registered using the mobile component of the tibial jig. During registration, the system confirms the orientation of the mobile component of the jig relative to the tibia using the differentials between the outputs of the accelerometers.

After the registration is complete, the display console provides dynamic numerical measurements of the alignment of the cutting block with respect to the mechanical axis in both the coronal and sagittal planes. The surgeon selects the acceptable resection angles before pinning the cutting block.

Postoperatively radiographs were done and the lower extremity mechanical axis, tibial component varus/valgus alignment, and femoral component varus/valgus alignment were digitally measured. The lower extremity mechanical axis was defined as the angle formed between a line drawn from the center of the femoral head, to central and most distal point of the inter-condylar notch of the femur, and a second line drawn between the centre of the tibial plateau and the centre of the tibial plafond.

The knee align display console provides real-time feedback of its orientation relative to the hip centre of rotation (both varus/valgus and flexion/extension). 
Preoperatively, standing anteroposterior (AP) and lateral knee radiographs were obtained for each patient, from which the lower extremity anatomic tibio-femoral axis, the mechanical axis (valgus/varus alignment) were measured as a part of the pre-operative preparation. Patient will then undergo the surgery.

Post operatively standing AP hip-to-ankle radiographs and standing lateral knee radiographs were obtained from which the tibial component varus/valgus, femoral component varus/valgus, and lower extremity mechanical alignment were digitally measured. For all AP radiographs, care was taken to ensure the patellae were facing forward to control for rotation.

The number of "outliers" in each, defined as a tibial or femoral component alignment outside of $3^{\circ}$ of perpendicular to the mechanical axis in the coronal plane and an overall lower extremity mechanical alignment outside of $3^{\circ}$ of a neutral mechanical axis, was determined. For individual component alignment, a deviation of greater than $3^{\circ}$ from neutral mechanical axis was selected as an "outlier" based on prior studies assessing the importance of overall, lower extremity alignment in TKA (Figures 3 and 4).

\section{Statistical methods}

Data will be entered in microsoft excel program and analysed by SPSS version 21. All the qualitative data are depicted perpendicular ( $\mathrm{a}>30$ or, $\mathrm{b}>30$ ) was selected as an "outlier" based on prior studies assessing the accuracy of component alignment in TKA. Similarly, a deviation of greater than $3^{\circ}$ from a as frequencies and percentage. All the quantitative data are depicted as mean \pm SD.

\section{RESULTS}

Demographic data are shown in Table 1. Among the 25 knees operated 2 cases were bilateral TKR ( 4 cases). In case of bilateral TKR, the $2^{\text {nd }}$ knee arthroplasty is done after 2 weeks of the initial surgery. The average age of the candidates that participated was 67.44 years (54-80 years). In the 23 candidates 4 were males and 19 were females. In the 25 knees operated 22 knees were in varus deformity and 3 were in valgus deformity (Table 1). The mean lower extremity mechanical axis of the knees in varus were $8.48 \pm 0.67^{\circ}$ and valgus knees were $-8.26 \pm 0.20^{\circ}$. Intraoperatively the average tourniquet time was 62 minutes (59 to 66 minutes). The target intraoperative tibial and femoral coronal alignment (varus/valgus) was $0^{\circ}$. Postoperatively, the average lower extremity mechanical alignment was $-1.46 \pm 0.49^{\circ}$ in those knees with a preoperative varus deformity and $-1.73 \pm 0.36^{\circ}$ in those knees with a preoperative valgus deformity. Overall, the mean postoperative lower extremity alignment was $1 \pm 0.39^{\circ}$, with $94.8 \%$ of patients having an alignment within $3^{\circ}$ of a neutral mechanical axis.

Table 1: Demographics and preoperative assessment.

\begin{tabular}{|ll|}
\hline Demographic & Value \\
\hline Age (years) & $62.5(45-80)$ \\
\hline \multirow{2}{*}{ Sex } & Males- 4 \\
\cline { 2 - 2 } & Females- 19 \\
\hline
\end{tabular}

Table 2: Comparison of the preoperative and postoperative lower extremity mechanical alignments, in addition to the tibial component positioning, for both the preoperative varus and valgus knees. note the negative values correspond to valgus alignment.

\begin{tabular}{|lllllll|}
\hline $\begin{array}{l}\text { Preop } \\
\text { deformity }\end{array}$ & Preop MA & Post op MA & $\begin{array}{l}\text { Tibial } \\
\text { component } \\
\text { alignment }\end{array}$ & $\begin{array}{l}(\%) \text { of tibial } \\
\text { component, } \\
90^{\circ} \pm 2^{\circ} \text { to MA }\end{array}$ & $\begin{array}{l}\text { Femoral } \\
\text { component } \\
\text { alignment }\end{array}$ & $\begin{array}{l}(\%) \text { of } \\
\text { femoral } \\
\text { component, } \\
90^{\circ} \pm 2^{\circ} \text { to } \mathbf{M A}^{-}\end{array}$ \\
\hline $\begin{array}{l}\text { Varus } \\
(\mathbf{n = 2 2})\end{array}$ & $8.48 \pm 0.67^{0}$ & $-1.46 \pm 0.49^{0}$ & $-1.29 \pm 0.39^{0}$ & 95.45 & $-1.60 \pm 0.40^{0}$ & 90.90 \\
\hline $\begin{array}{l}\text { Valgus } \\
(\mathbf{n = 3})\end{array}$ & $-8.26 \pm 0.20^{0}$ & $-1.73 \pm 0.36^{0}$ & $-1.43 \pm 0.38^{0}$ & 100 & $-1.4 \pm 0.35^{0}$ & 100 \\
\hline
\end{tabular}

Postoperatively, the overall mean radiographic varus/ valgus alignment of the tibial components was $1.33 \pm 0.400$. The mean absolute difference between the intraoperative goal of $0^{\circ}$ (or perpendicular to the mechanical axis) and the actual postoperative tibial component alignment measured on radiographs was $1.11^{\circ} \pm 0.48^{\circ}$, with $96.0 \%$ positioned within $2^{\circ}$ and $100 \%$ within $3^{\circ}$ of the intraoperative goal. With regard to the femoral component, the overall mean radiographic varus/valgus alignment was also $-1.58 \pm 0.40 .0$. The mean absolute difference between the intraoperative goal of $0^{\circ}$ and the actual postoperative femoral component alignment measured on radiographs was $1.07^{\circ} \pm 0.49^{\circ}$, with $92.0 \%$ positioned within $2^{\circ}$ and $100 \%$ within $3^{\circ}$ of the intraoperative goal.

\section{DISCUSSION}

TKA has been considered as a successful procedure in treating osteoarthritis of knee and it has proved to have long term success rate. Malalignment is one of the important causes for the failure of TKA surgeries. ${ }^{7}$ Numerous various comparative studies have pointed out CAS techniques to be superior to conventional IM and EM guides in TKA with regard to component alignment but 
drawbacks like increased operative times, learning curve, and costs have limited its acceptance. ${ }^{5}$

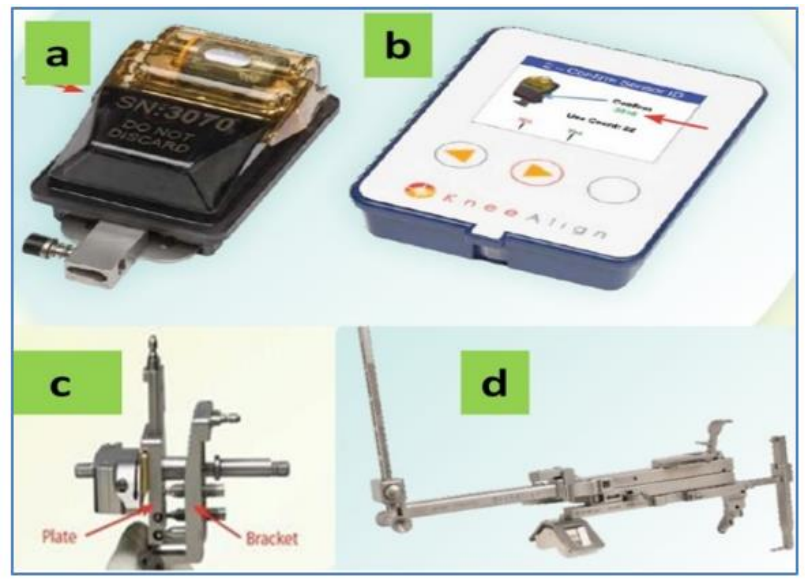

Figure 1: Parts of a hand held navigation system, asensor, b-display console, c-femoral jig, d-tibial jig.

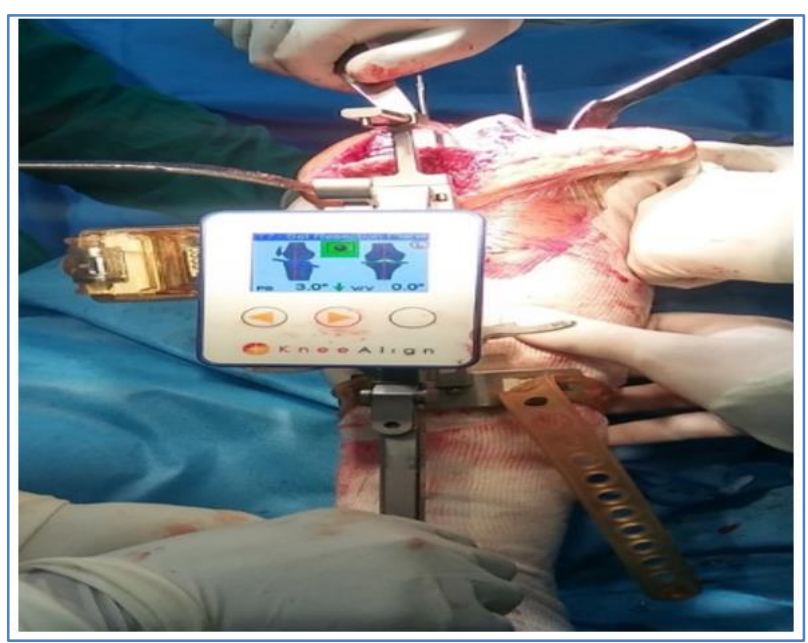

Figure 2: Assembled femoral jig with a sensor and a display console showing real time feedback.

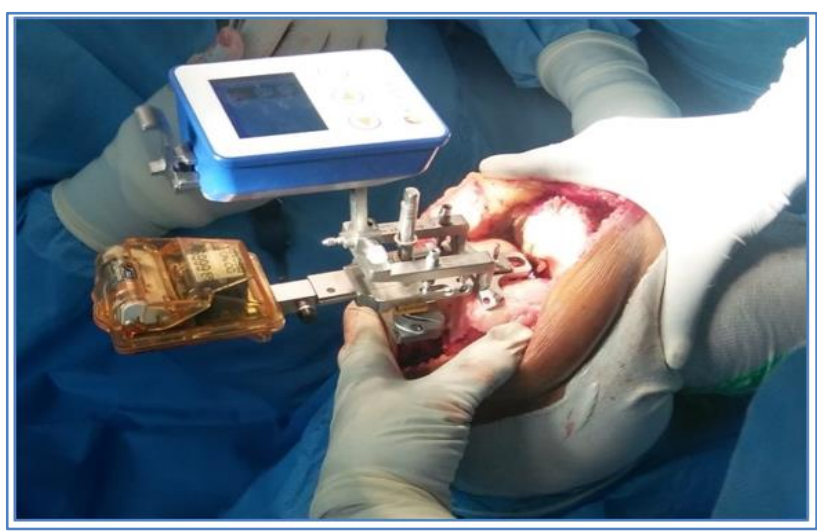

Figure 3: Assembled tibial jig with a sensor and a display console showing real time feedback.

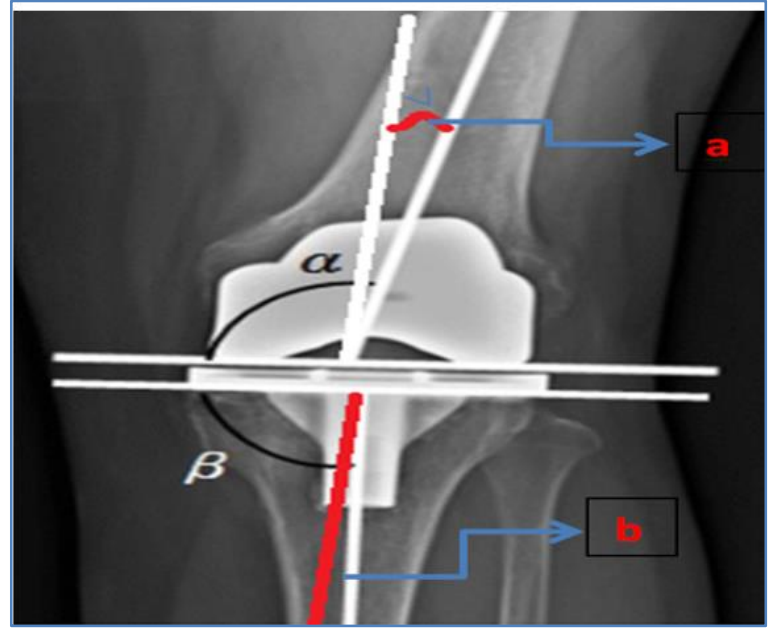

Figure 4: Post-operative radiographic measurements $\alpha$ : femoral component varus/valgus, $\beta$ : tibial component varus/valgus, $a=90-\alpha, b=90-\beta$.

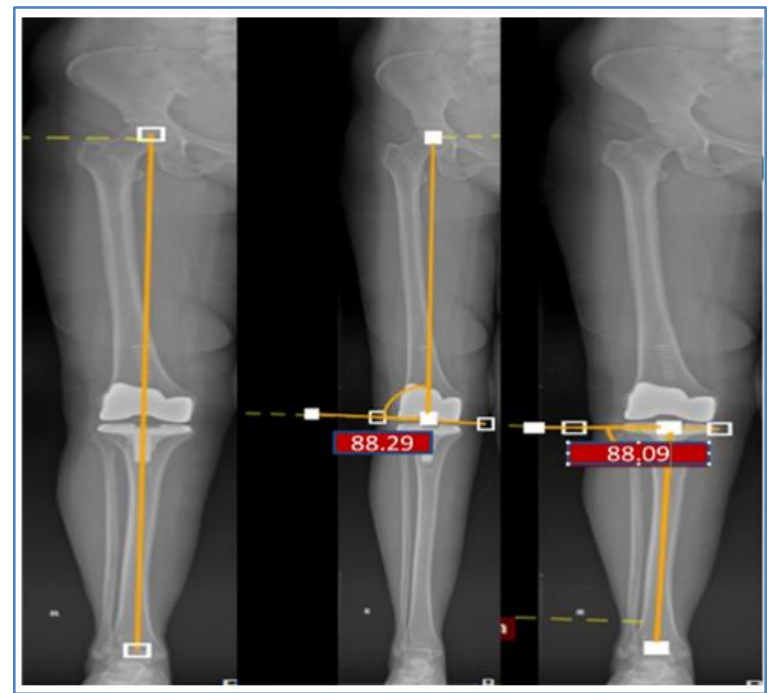

Figure 5: From right to left showing overall mechanical axis, femoral component varus/valgus; tibial component varus /valgus.

The purpose of this study was to assess the accuracy of a portable, accelerometer-based navigation system in achieving a distal femoral and tibial -component alignment perpendicular to the mechanical axis in the coronal plane. Berend et al. studied tibial component failure mechanisms in 3152 TKA and concluded that tibial malalignment is a strong predictor for revision and most importantly $>3^{\circ}$ of varus in the tibial component increased the odds of implant failure 17-fold. ${ }^{2}$ Paratte et al, studied equal survival of TKA implants at 15 years whether the alignment met the $\pm 3^{\circ}$ criteria or not. They state, until additional data can be generated to more accurately determine the ideal postoperative limb alignment... a neutral mechanical axis remains a reasonable target and should be considered as the standard for comparison. ${ }^{8}$ 
This study demonstrates that the knee align system is very much precise in performing the distal femoral and proximal tibial resection in TKA, as $92 \%$ of the femoral components and $96 \%$ of the tibial components were aligned within $2^{\circ}$ of perpendicular to the femoral and tibial mechanical axis, and $94.8 \%$ of patients had an overall mechanical alignment within $3^{\circ}$ of a neutral mechanical axis. $100 \%$ of both tibial and femoral components were placed within 3 degrees of the perpendicular (Table 2).

Even though these results are promising, there are several limitations to this study. First, this study presents a case series of patients treated by a single surgeon. Secondly the sample size is low and the study duration is short and it is not a randomized study. There is no control group using conventional IM or EM guides to which these patients are usually compared. Instead, these data can only be compared with historical controls. No data are presented with regard to the learning curve required to use the device. However, the device is simple to use, as minimal steps are required to perform the distal femoral and proximal tibial cutting blocks.
The device is portable, and the large computer consoles and extra pin sites/arrays necessary for most CAS systems are not required. In addition, the knee align femoral and tibial jig and cutting block are similar in design to that of a conventional IM alignment guide for the femur (minus the IM rod), thereby providing familiarity to a surgeon using the device for the first time. Lastly, although standing AP hip-to-ankle radiographs were used to measure the femoral component alignment, it could be argued that computed tomography is more accurate in determining component positioning and also enable measurements of flexion/extension of the femoral components. However, computed tomography has several disadvantages, including radiation exposure, cost, and artefact surrounding the implants, and it is not used for routine follow-up in the clinical setting.

The similar studies conducted by Iorio et al and Nam et al on the component alignment in TKR using accelerometer based hand held navigation system which had a bigger sample size (up to 151) and longer study period showed similar results as our study (Table 3). ${ }^{9,11}$

Table 3: Comparison of similar studies with our study showing similar results.

\begin{tabular}{|c|c|c|c|}
\hline & $\begin{array}{l}\text { (\%) Post op mechanical } \\
\text { axis }\end{array}$ & $\begin{array}{l}(\%) \text { of tibial component, } \\
90^{\circ} \pm 2^{\circ} \text { to MA (\%) }\end{array}$ & $\begin{array}{l}\text { (\%) of femoral component, } \\
90^{\circ} \pm 2^{\circ} \text { to MA (\%) }\end{array}$ \\
\hline Our study (n=25) & 94.3 & 96.0 & 92.0 \\
\hline Nam et $\mathrm{al}^{\mathbf{1}}(\mathrm{n}=48)$ & 93.8 & - & 95.8 \\
\hline Nam et $a^{12}(n=151)$ & 93.8 & 95.3 & - \\
\hline Iorio et $\mathbf{a l}^{9}(\mathrm{n}=50)$ & 94 & 96 & - \\
\hline
\end{tabular}

MA: mechanical axis; $n$ : the sample size.

\section{CONCLUSION}

The knee align system effectively pools the advantages of large-console CAS systems, while avoiding the disadvantages of conventional IM femoral alignment systems. This study demonstrates that portable, accelerometer-based navigation is highly accurate in positioning the femoral and tibial component in TKA.

\section{Declarations}

The study was conducted among the patients admitted for only primary knee arthroplasty using portable accelerometer based hand held navigation, in the department of orthopaedics. It does not require any animal study. The patients will be subjected to investigations which are relevant and routinely done as a part of patient management after taking written informed consent.

Funding: No funding sources

Conflict of interest: None declared

Ethical approval: The study was approved by the Vydehi institutional ethics committee

\section{REFERENCES}

1. Azar MF, Canale S, Beaty HJ. Campbell's Operative Orthopaedics. 13 ${ }^{\text {th }}$ ed. Tennessee: Elsevier; 2016; 413-458.

2. Berend M, Ritter M, Keating E, Faris P, Meding J. Tibial component failure mechanisms in total knee replacement. J Arthroplasty. 2004;19(2):262.

3. Hafez M, Moholkar K. Patient-specific instruments: advantages and pitfalls. SICOT-J. 2017;3:66.

4. Shen C, Tang Z, Hu J, Zou G, Xiao R, Yan D. Patient-Specific Instrumentation Does Not Improve Accuracy in Total Knee Arthroplasty. Orthopedics. 2015;38(3):178-88.

5. Xie C, Liu K, Xiao L, Tang R. Clinical Outcomes After Computer-assisted Versus Conventional Total Knee Arthroplasty. Orthopedics. 2012;35(5):647-53.

6. Dennis D, Channer M, Susman M, Stringer E. Intramedullary versus extramedullary tibial alignment systems in total knee arthroplasty. J Arthroplasty. 1993;8(1):43-7.

7. Sharkey P, Hozack W, Rothman R, Shastri S, Jacoby S. Why Are Total Knee Arthroplasties Failing Today. Clinical Orthop Related Res. 2002;404:7-13.

8. Parratte S, Pagnano M, Trousdale R, Berry D. Effect of Postoperative Mechanical Axis Alignment on the Fifteen-Year Survival of Modern, Cemented Total 
Knee Replacements. J Bone Joint Surgery-American Vol. 2010;92(12):2143-9.

9. Iorio R, Mazza D, Drogo P, Bolle G, Conteduca F, Redler A, et al. Clinical and radiographic outcomes of an accelerometer-based system for the tibial resection in total knee arthroplasty. Int Orthop. (2014);39(3):461-6.

10. Bugbee W, Kermanshahi A, Munro M, Cauley J Copp S. Accuracy of a hand-held surgical navigation system for tibial resection in total knee arthroplasty. Knee. 2014;21(6):1225-8.

11. Nam D, Cody E, Nguyen J, Figgie M, Mayman D. Extramedullary Guides Versus Portable, Accelerometer-Based Navigation for Tibial
Alignment in Total Knee Arthroplasty: A Randomized, Controlled Trial: Winner of the 2013 HAP PAUL Award; 2014.

12. Nam D, Nawabi D, Cross M, Heyse T, Mayman D. Accelerometer-Based Computer Navigation for Performing the Distal Femoral Resection in Total Knee Arthroplasty. J Arthroplasty. 2012;27(9):1717-22.

Cite this article as: Hiranyakumar S, Nadagouda S, Harikrishnan V. A study on radiological outcomes of accelerometer based hand held navigation system in total knee arthroplasty. Int J Res Orthop 2020;6:556-61. 